\title{
Effect of Porphyromonas gingivalis lipopolysaccharide on calcification of human umbilical artery smooth muscle cells co-cultured with human periodontal ligament cells
}

\author{
JING LI ${ }^{1,2^{*}}$, JING DENG ${ }^{1,2^{*}}$, SHUXIAN SHANG ${ }^{1,2}$, GUIRONG LIU $^{3}$, WENBIN SONG ${ }^{1,2}$, \\ PEI SUN ${ }^{1,2}$, WENJING JIANG ${ }^{1,2}$ and KEQING PAN ${ }^{1,2}$ \\ ${ }^{1}$ Department of Stomatology, The Affiliated Hospital of Qingdao University; \\ ${ }^{2}$ School of Stomatology of Qingdao University, Qingdao, Shandong 266003; \\ ${ }^{3}$ Department of Stomatology, Qingdao Municipal Hospital, Qingdao, Shandong 266011, P.R. China
}

Received December 4, 2020; Accepted March 11, 2021

DOI: $10.3892 / \mathrm{etm} .2021 .10087$

\begin{abstract}
Periodontitis is an independent risk factor for coronary heart disease. Porphyromonas gingivalis lipopolysaccharide (Pg-LPS) was considered to be one of the main virulence factors. In addition, vascular smooth muscle cells transform into osteoblast-like cells in an arterial calcification process under chronic inflammatory conditions. The present study aimed to determine the calcification induced by Pg-LPS in human umbilical artery smooth muscle cells (HUASMCs) co-cultured with human periodontal ligament cells (HPDLCs). An in vitro co-culture system was established using Transwell inserts. HUASMC proliferation and alkaline phosphatase (ALP) activity were measured with a Cell Counting Kit- 8 and an ALP kit, respectively. Calcium nodule formation was detected using alizarin red S staining. The effects of Pg-LPS on the mRNA expression of the calcification genes of ALP, core-binding factor $\alpha 1$ (Runx2) and bone sialoprotein (BSP) were assessed using reverse transcription-quantitative PCR. The results indicated that Pg-LPS increased HUASMC proliferation and ALP activity. Furthermore, among all of the groups, calcium nodule formation was most extensive in co-cultured cells in the mineralization-inducing medium containing Pg-LPS. In addition, the expression of specific osteogenic genes (Runx2, ALP and BSP) significantly increased in the presence of Pg-LPS and mineralization-inducing medium, which was further enhanced in co-culture with HPDLCs. In conclusion, co-culture with HPDLCs increased the effect of Pg-LPS to stimulate the calcification of HUASMCs. It was suggested that besides the inflammation, periodontitis may
\end{abstract}

Correspondence to: Dr Keqing Pan, Department of Stomatology, The Affiliated Hospital of Qingdao University, 16 Jiangsu Road, Qingdao, Shandong 266003, P.R. China

E-mail: pankeqing77@sina.com

Key words: co-culture, periodontitis, Porphyromonas gingivalis lipopolysaccharide, calcification promote the occurrence of vascular calcification. The study indicated that periodontal treatment of subgingival scaling to reduce and/or control Porphyromonas gingivalis may decrease the occurrence or severity of vascular calcification.

\section{Introduction}

Periodontal disease is a chronic and non-specific infectious disease of tooth-supporting periodontal tissues mainly caused by Gram-negative bacterial infection. Periodontal ligament (PDL) is a specialized soft connective tissue that connects the tooth root surface with the alveolar bone socket. It consists of different cell populations, including endothelial cells, fibroblasts, epithelial cells, osteoblasts, the rest of Malassez and cementoblasts (1). Porphyromonas gingivalis (Pg), a Gram-negative bacterial species, has been extensively studied as one of the putative periodontal pathogens with sufficient evidence (2). Lipopolysaccharide (LPS) is the major pathogenic factor on the surface of Gram-negative bacteria. LPS from Pg (Pg-LPS), considered as one of the main virulence factors of Pg, may induce inflammatory cytokine secretion by periodontal cells $(3,4)$, damaging periodontal tissue and leading to the resorption of the alveolar bone (5-7).

Periodontitis is an independent risk factor for coronary heart disease (8). As a significant risk factor for cardiovascular disease, vascular calcification is commonly present in atherosclerosis (9). In the past, vascular calcification was considered a passive regulator of aging-related diseases; while it is now generally recognized as an actively regulated bone-like formation process with the transformation of vascular smooth muscle cells(VSMCs) into osteoblast-like cells with the expression of osteogenesis-related proteins (10). It has been indicated that vascular calcification is correlated with inflammation and is recognized as a procedural osteogenesis process initiated by endovascular inflammatory factors (11). The phenotype of VSMCs is transformed into osteoblast-like cells in the arterial calcification process under chronic inflammatory conditions (12). Preliminary studies of our group suggested that Pg-LPS promoted the abnormal proliferation and alkaline phosphatase (ALP) activity of rat aortic smooth muscle 
cells; the expression of the calcification-related genes ALP, core-binding factor $\alpha 1$ (Runx2), bone sialoprotein (BSP) and osteopontin (OPN) increased, further suggesting that Pg-LPS had a specific effect on calcification $(13,14)$.

However, the microenvironment of the human body is complex, where the effects of VSMCs on vascular calcification in the setting of periodontal disease remained to be fully determined. In the present study, a three-dimensional co-culture model of human periodontal ligament cells (HPDLCs) and human umbilical artery smooth muscle cells (HUASMCs) was established by using Transwell inserts to simulate the complex microenvironment including the coexistence of periodontitis and vascular calcification. The present study explored the effects of Pg-LPS on the proliferation, ALP activity, formation of calcified nodules and mRNA expression of related calcification genes (ALP, Runx2 and BSP) in HUASMCs under co-culture conditions.

\section{Materials and methods}

Cell culture and characterization. The present study was approved by the Medical Ethics Committee of the Affiliated Hospital of Qingdao University (Qingdao, China; no. QYFYWZLL26135). Written informed consent was obtained from each patient or from the parents/guardians of those participants who were minors. To obtain HPDLCs, premolars (healthy orthodontic tooth extractions) without caries, periodontal disease nor periapical periodontitis freshly extracted between December 2017 and December 2018 were selected. The patients (20 males and 20 females) were between 12 and 25 years old. All the cells from 60 teeth were pooled for different assays. The primary culture of HPDLCs was established according to a method described previously (15). First, the cells were cultured in Dulbecco's Modified Eagle Medium (DMEM; HyClone; Cytiva) containing 20\% fetal bovine serum (FBS) (Gibco; Thermo Fisher Scientific, Inc.) in an atmosphere under 5\% $\mathrm{CO}_{2}$ at $37^{\circ} \mathrm{C}$. The morphology and growth of the cells were observed. After the cells reached $80 \%$ confluency, they were passaged by trypsinization (Beijing Solarbio Science \& Technology Co., Ltd.). After passaging, the medium was replaced with medium containing 10\% FBS. The third-generation cells were identified by vimentin (cat. no. PB9359; Boster Biological Technology) and keratin (cat. no. BM0031; Boster Biological Technology) staining with the streptavidin-biotin complex (cat. no. SA1021, Boster Biological Technology) immunohistochemical method. Cells in the third to fifth generation with good viability were used for the subsequent experiments.

Primary HUASMCs (cat. no. 8030) were purchased from ScienCell Research Laboratories, Inc. and subcultured in DMEM (Gibco; Thermo Fisher Scientific, Inc.) supplemented with 10\% FBS (Gibco; Thermo Fisher Scientific, Inc.) at $37^{\circ} \mathrm{C}$ with $5 \% \mathrm{CO}_{2}$ after recovery. The third generation HUASMCs were prepared on cell slides at $1 \times 10^{4}$ cells $/ \mathrm{ml}$ for $\alpha$-smooth muscle actin ( $\alpha$-SMA; cat. no. BS70000; Bioworld Technology, Inc.) immunofluorescence staining. The cells in the exponential growth phase from the third generation to the eighth generation were used for the subsequent experiments.

Co-culture. HUASMCs were seeded into six-well microplates (Corning, Inc.) in DMEM containing 10\% FBS at
$1 \times 10^{4}$ cells $/ \mathrm{ml}$. After $4 \mathrm{~h}$, the cell took adhesion at $37^{\circ} \mathrm{C}$ with $5 \% \mathrm{CO}_{2}$, fully expanded to a confluency of $70 \%$. The medium was then refreshed and the HPDLCs were seeded into Transwell inserts in DMEM containing 10\% FBS, at $1 \times 10^{4}$ cells $/ \mathrm{ml}$. The non-contact HPDLCs-HUASMCs co-culture system was established using Transwell inserts (Corning, Inc.) with a pore size of $0.4 \mu \mathrm{m}$ (16). Co-cultured cells were not in direct contact but were able to interact with each other via the culture fluid for $48 \mathrm{~h} 37^{\circ} \mathrm{C}$ with $5 \% \mathrm{CO}_{2}$.

Cell viability assay. The cells were divided into four experimental groups as follows: i) HUASMC group, which was cultured normally (group C); ii) HPDLCs-HUASMCs co-culture group (group CO); iii) HUASMCs group cultured with Pg-LPS (group CP; InvivoGen); and iv) HPDLCs-HUASMCs co-culture group cultured with Pg-LPS (group CO-P). In addition, the four groups as above were set up in calcification-inducing culture medium (groups $\mathrm{C}-\mathrm{C}, \mathrm{CO}-\mathrm{C}, \mathrm{CP}-\mathrm{C}, \mathrm{CO}-\mathrm{CP}$, respectively). The final concentration of Pg-LPS added in the experiment was $1 \mu \mathrm{g} / \mathrm{ml}(13,17)$. The cells were inoculated according to the experimental groups. HUASMCs were inoculated in 24-well microplates (Corning, Inc.) and Transwell inserts containing HPDLCs were placed on top of the 24-well microplates to establish the HPDLCs-HUASMCs co-culture system. HUASMCs alone were first cultured without HPDLCs in DMEM containing $10 \%$ FBS for $24 \mathrm{~h}$, followed by synchronization in serum-free medium for $12 \mathrm{~h}$, and subsequently, culture was performed for $48 \mathrm{~h}$ according to each group's treatment requirements. The viability of the HUASMCs as a confluence of 70-90\% was measured by a Cell Counting Kit-8 (Dojindo Molecular Technologies, Inc.). For the cell viability assay, $50 \mu \mathrm{l} \mathrm{CCK-8}$ solution was added to each well containing $500 \mu 1$ medium (volume ratio, $10 \%$ ) for different doses or varying time points; air bubbles were avoided. The plates were incubated at $37^{\circ} \mathrm{C}$ with $5 \% \mathrm{CO}_{2}$ for $2 \mathrm{~h}$, according to the manufacturer's protocol. The absorbance was measured with a spectrophotometer at a wavelength of $450 \mathrm{~nm}$. The experimental results were expressed as the optical density.

ALP assay. The effects of normal medium or calcification-inducing culture medium on ALP activity of HUASMCs were measured by an ALP kit (cat. no. A059-2-2, Nanjing Jiancheng Bioengineering Institute) according to the manufacturer's protocol. The experimental groups and culture methods were the same as those in the above experiment; the cells for the relevant groups were inoculated and cultured for $48 \mathrm{~h}$ at $37^{\circ} \mathrm{C}$ with $5 \% \mathrm{CO}_{2}$. The ALP activity and protein concentration in the supernatant were measured using a microplate reader at a wavelength of $520 \mathrm{~nm}$. The results were normalized to the total protein content per sample using a bicinchoninic acid protein assay kit (Pierce; Thermo Fisher Scientific, Inc.) and expressed in fold induction of ALP activity (18).

Calcification-inducing culture. Calcification-inducing culture medium was prepared as follows: $\beta$-glycerophosphate, vitamin $C$ and dexamethasone (all from Sigma-Aldrich; Merck KGaA), with final concentrations of 1,50 and $10 \mathrm{mM}$, respectively, were added to DMEM containing 5\% FBS. 
Table I. Primers used for real-time PCR analyses.

Gene/sequence ( $5^{\prime}$ to $\left.3^{\prime}\right)$

Product length (bp)

GenBank accession no.

Alkaline phosphatase
Forward, AGAATCTGGTGCAGGAATGG
Reverse, AGGCTCAAAGAGACCCATGA
Runx2
Forward, GTGGACGAGGCAAGAGTTTC
Reverse, TTCCCGAGGTCCATCTACTG

Bone sialoprotein

122

NM_001632.4

Forward, CGGAGGAGACAATGGAGAAG

Reverse, CCACCATTTGGAGAGGTTGT

GAPDH

138

NM_002046.6

Forward, GTCTCCTCTGACTTCAACAGCG

Reverse, ACCACCCTGTTGCTGTAGCCAA

Runx2, core-binding factor $\alpha 1$.

This part of the experiment consisted of eight groups, which were all cultured in Transwell plates at $1 \times 10^{4}$ cells $/ \mathrm{ml}$. In four groups, the treatment was performed with normal culture medium (groups $\mathrm{C}, \mathrm{CO}, \mathrm{CP}$ and $\mathrm{CO}-\mathrm{P}$ ) whereas the four other groups were cultured as follows: HUASMCs incubated with the calcification-inducing culture medium (group CC) or with Pg-LPS-containing calcifying culture medium (group C-CP); HPDLCs-HUASMCs co-cultured cultured in calcifying medium (group CO-C) or with Pg-LPS-containing calcified medium (group CO-CP). The cultured cells were inoculated to set up the groups and the medium was changed every 3 days up to 21 days.

Expression of calcification-related factors (ALP, Runx2 and $B S P$ ) determined by reverse transcription-quantitative $(R T-q) P C R$. The different groups were set up according to the pre-set experimental conditions and cultured for $48 \mathrm{~h}$. The total cellular RNA was extracted using TRIzol ${ }^{\circledR}$ (Thermo Fisher Scientific, Inc.) according to the instructions of the manufacturer. The concentration and purity of RNA were measured using NanoDrop spectrophotometer (Thermo Fisher Scientific, Inc.). For each group, $1.0 \mu \mathrm{g}$ of RNA was reversely transcribed into complementary (c) DNA in a $20-\mu 1$ reaction system using PrimerScript ${ }^{\mathrm{TM}}$ RT reagent Kit (cat. no. RR047A, Takara Bio, Inc.) a kit with gDNA Eraser. The total reaction mixture was incubated at $50^{\circ} \mathrm{C}$ for $15 \mathrm{~min}$ and $85^{\circ} \mathrm{C}$ for $5 \mathrm{sec}$. The PCR amplification reaction was performed on $1.0 \mu \mathrm{l}$ of cDNA in a $20-\mu \mathrm{l}$ reaction system using TB Green ${ }^{\text {TM }}$ Premix Ex Taq ${ }^{\text {TM }}$ II(Tli RNaseH Plus) (cat. no. RR820A; Takara Bio, Inc.) on a 7500 qPCR system (Applied Biosystems; Thermo Fisher Scientific, Inc.). The thermocycling conditions were as follows: Initial denaturation at $95^{\circ} \mathrm{C}$ for $10 \mathrm{~min}$; followed by 40 cycles of $95^{\circ} \mathrm{C}$ for $1 \mathrm{~min}$ and $60^{\circ} \mathrm{C}$ for $1 \mathrm{~min}$. Specific primer sequences for ALP, Runx 2, BSP and GAPDH were designed and synthesized by Sangon Biotech, Co., Ltd. and their sequences are provided in Table I. The GAPDH gene expression levels were calculated using the $2^{-\Delta \Delta \mathrm{Cq}}$ method (19).
Calcification-specific alizarin red $S$ staining and quantitative analysis of calcified nodules. After culturing for 3 weeks, the Transwell inserts were removed and the culture medium was discarded, followed by rinsing twice with pre-warmed PBS, addition of $4 \%$ paraformaldehyde and keeping at room temperature for $30 \mathrm{~min}$. The cells were rinsed with distilled water twice. Subsequently, $1 \%$ alizarin $\mathrm{S}$ red dye liquor (Beijing Solarbio Science \& Technology Co., Ltd.) was added to cover the culture plate's bottom, with microscopic observation and gentle shaking by hands and keeping at room temperature for $15 \mathrm{~min}$. After rinsing with distilled water four times, the solution was gently shaken for $5 \mathrm{~min}$ and incubated at $-20^{\circ} \mathrm{C}$ for $10 \mathrm{~min}$.

The results were observed under an inverted microscope. The quantitative analysis method for calcium nodules was according to Gregory et al (20), with $150 \mu \mathrm{l}$ of the liquid pipetted from each tube and transferred to a 96 -well microplate (Corning, Inc.) to determine the absorbance at a wavelength of $405 \mathrm{~nm}$ using a microplate reader.

Statistical analysis. Values are expressed as the mean \pm standard deviation from at least 3 independent experiments. One-way analysis of variance was performed for comparisons among the groups and intergroup comparisons were performed using Bonferroni's correction using SPSS statistical software (version 22.0; IBM Corp.). $\mathrm{P}<0.05$ was considered to indicate a statistically significant difference.

\section{Results}

Characterization of the cells. The HPDLCs were positive and negative for anti-vimentin (Fig. 1A) and anti-keratin staining (Fig. 1B), respectively. $\alpha$-SMA was highly expressed in HUASMCs (Fig. 1C). The HPDLCs in the Transwell inserts were adherent and they exhibited long spindle or radial arrangements after being inoculated for $\sim 12 \mathrm{~h}$ (Fig. 2A). The HUASMCs in the culture plate were adherent and were polygonal or spindle-shaped after inoculation for $16 \mathrm{~h}$ (Fig. 2B). 

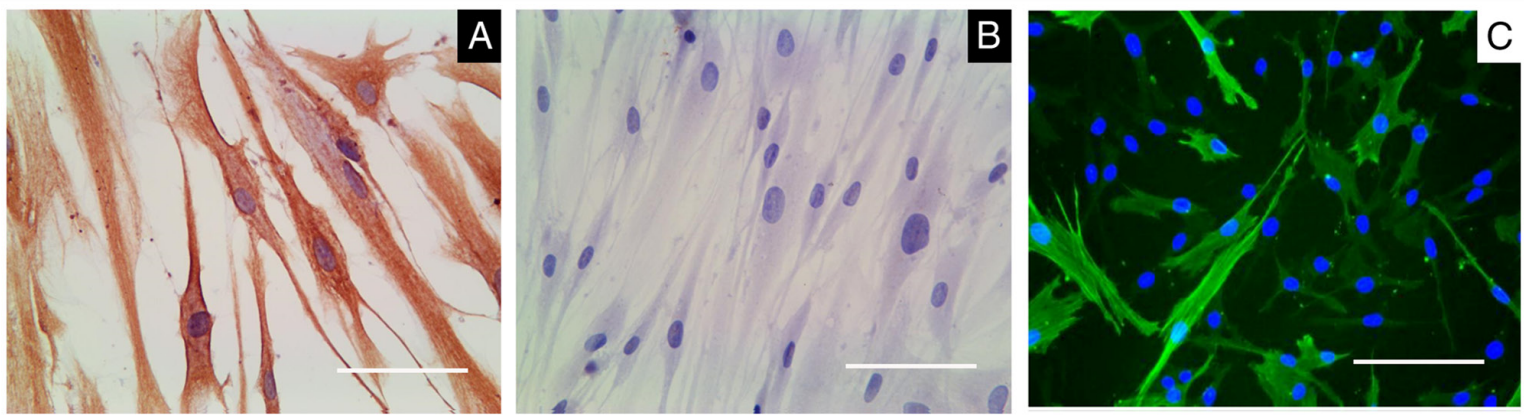

Figure 1. HPDLCs and HUASMCs were characterized. (A) HPDLCs were positive for anti-vimentin staining. (B) HPDLCs were negative for anti-keratin staining. (C) HUASMCs expressed $\alpha$-smooth muscle actin. The DAPI staining image is superimposed with green fluorescence, with $\alpha$-SMA being fluorescent green and the nuclei blue (scale bars, $100 \mu \mathrm{m}$ ). HUASMCs, human umbilical artery smooth muscle cells; HPDLCs, human periodontal ligament cells.
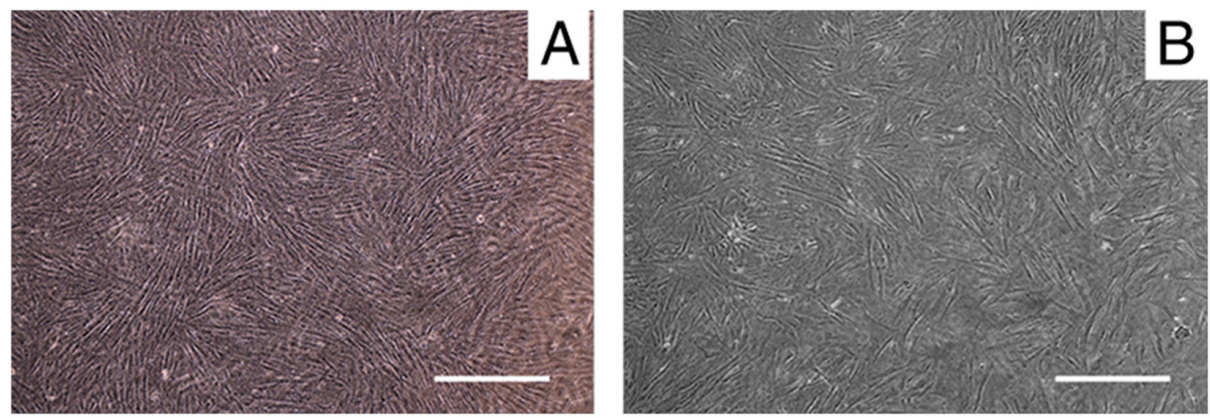

Figure 2. HPDLC-HUASMC co-culture system was established with Transwell inserts. (A) HPDLCs, with micropores in the porous membrane visible (B) HUASMCs, exhibiting a radial spindle-shaped arrangement when attached to the plate (scale bars, $20 \mu \mathrm{m}$ ). HUASMCs, human umbilical artery smooth muscle cells; HPDLCs, human periodontal ligament cells.

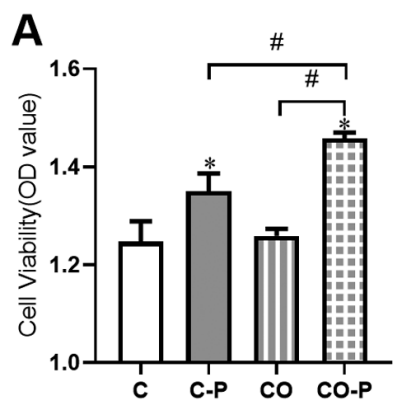

\section{C: HUASMCs}

CP: HUASMCs+Pg-LPS

CO: PDLCs-HUASMCs

CO-P:PDLCs-HUASMCs+Pg-LPS

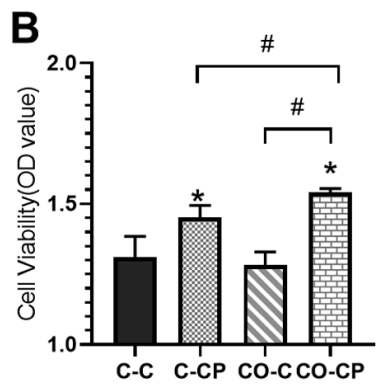

\author{
C-C: HUASMCs+ \\ calcification-inducing culture medium \\ C-CP: \\ HUASMCs+Pg-LPS+calcification-indu \\ cing culture medium \\ CO-C: \\ PDLCs-HUASMCs+calcification-induc \\ ing culture medium \\ CO-CP: \\ PDLCs-HUASMCs+Pg-LPS+calcificat \\ ion-inducing culture medium
}

Figure 3. Cell viability. (A) Effect of Pg-LPS on HUASMC viability in the co-culture system. (B) Cell viability in HUASMCs treated with or without Pg-LPS in the calcified-inducing culture medium. Values are expressed as the mean \pm standard deviation of three independent experiments in each group. ${ }^{*} \mathrm{P}<0.05$ vs. HUASMCs (group C); ${ }^{\text {P }}<0.05$. HUASMCs, human umbilical artery smooth muscle cells; PDLCs, periodontal ligament cells; Pg-LPS, Porphyromonas gingivalis lipopolysaccharide; ALP, alkaline phosphatase.

Pg-LPS-stimulated HUASMC proliferation is increased in the co-culture system. The effect of Pg-LPS treatment for 48 $\mathrm{h}$ on HUASMCs to increase the proliferation of co-cultured HUASMCs was greater than that of monoculture HUASMCs and the difference was statistically significant. However, there was no significant difference in the proliferation of HUASMCs in mono- vs. co-culture without Pg-LPS stimulation (Fig. 3A). The trends for the co-culture system were similar in the calcification-inducing culture medium (Fig. 3B).

Effects of normal medium and calcification-inducing culture medium on ALP activity of HUASMCs. After Pg-LPS stimulation for $48 \mathrm{~h}$, the ALP activity in the supernatant of HUASMCs under co-culture conditions was enhanced and higher than that of the HUASMCs in monoculture, with a significant difference. However, there was no significant difference in the ALP activity in the supernatant of HUASMCs in mono- vs. co-culture without Pg-LPS stimulation (Fig. 4A). This phenomenon was similar in the groups with Pg-LPS stimulation as in the calcification-inducing culture medium. However, co-culturing the cells in calcification-inducing culture medium exhibited significantly higher ALP activity compared with HUASMCs in calcification-inducing culture medium, with a significant difference. (Fig. 4B).

Relative quantification of osteogenic gene induction by $P g$-LPS in HUASMCs in the co-culture system. The relative 

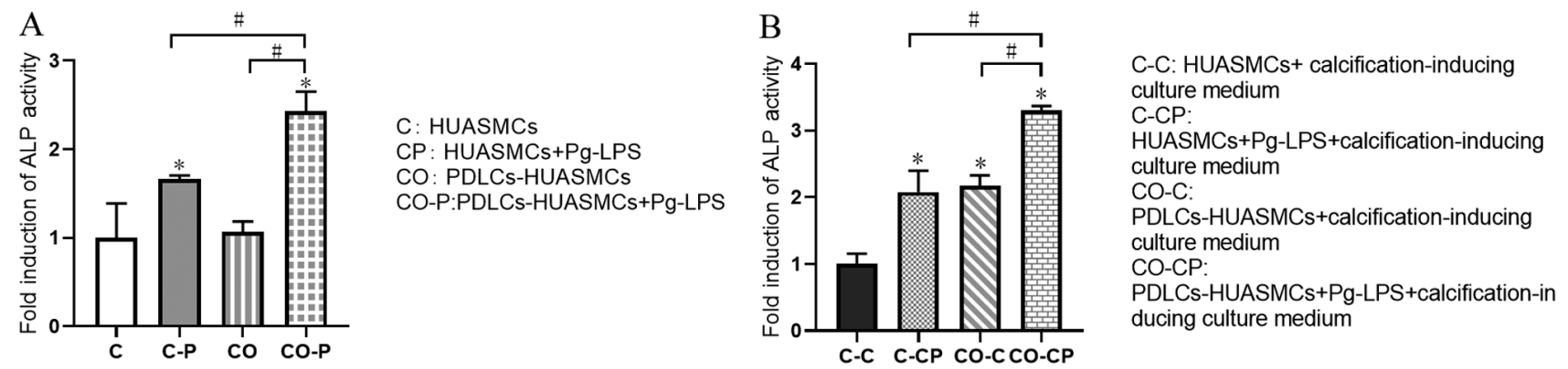

Figure 4. Induction of ALP activity. Effects of the normal medium (A) and the calcification-inducing culture medium (B) on ALP activity of HUASMCs and the HUASMCs-HPDLCs co-culture system. Values are expressed as the mean \pm standard deviation of three independent experiments in each group. ${ }^{*} \mathrm{P}<0.05$ vs. HUASMCs in calcification-inducing culture medium (group CC); ${ }^{*} \mathrm{P}<0.05$. HUASMCs, human umbilical artery smooth muscle cells; PDLCs, periodontal ligament cells; Pg-LPS, Porphyromonas gingivalis lipopolysaccharide; ALP, alkaline phosphatase; OD, optical density.
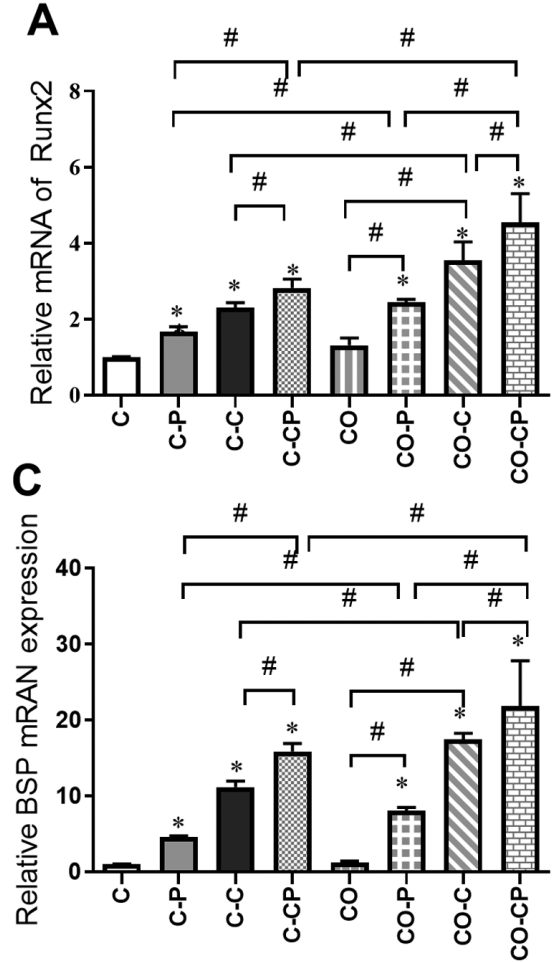

B

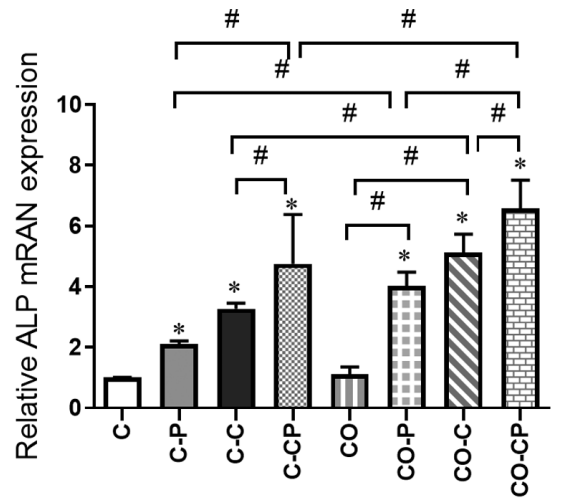

C: HUASMCS

C-P: HUASMCs+Pg-LPS

CO: PDLCs-HUASMCs

CO-P: PDLCs-HUASMCs+Pg-LPS

$C-C$ : HUASMCs+calcification-inducing culture medium

C-CP: HUASMCs+Pg-LPS+calcification-inducing culture medium

CO-C: PDLCs-HUASMCs+calcification-inducing culture medium

CO-CP: PDLCs-HUASMCs+Pg-LPS+calcification-inducing culture

medium

Figure 5. Induction of osteogenic gene transcription in HUASMCs. Transcript levels for the osteogenic genes (A) Runx2, (B) ALP and (C) BSP were determined at $48 \mathrm{~h}$ by reverse transcription-quantitative PCR and normalized to the level of GAPDH transcript. Values are expressed as the mean \pm standard deviation (n=3). The expression of Runx2, ALP and BSP was significantly higher in co-culture groups treated with Pg-LPS (1 $\mu \mathrm{g} / \mathrm{ml})$ and calcification-inducing medium than in the control groups. " $\mathrm{P}<0.05$ vs. HUASMCs (group C); "P<0.05. HUASMCs, human umbilical artery smooth muscle cells; PDLCs, periodontal ligament cells; Pg-LPS, Porphyromonas gingivalis lipopolysaccharide; ALP, alkaline phosphatase; Runx2, core-binding factor $\alpha 1$; BSP, bone sialoprotein; OD, optical density.

expression of calcification-related factors was detected by RT-qPCR in the different experimental groups after incubation for $48 \mathrm{~h}$. The expression of Runx 2, ALP and BSP was significantly higher in the groups with calcification induction medium $(\mathrm{C}-\mathrm{C}, \mathrm{C}-\mathrm{CP}, \mathrm{CO}-\mathrm{C}$ and $\mathrm{CO}-\mathrm{CP})$ than in the groups with normal culture medium $(\mathrm{C}, \mathrm{C}-\mathrm{P}, \mathrm{CO}$ and $\mathrm{CO}-\mathrm{P}$, respectively) and significantly higher in groups with Pg-LPS (C-P, $\mathrm{C}-\mathrm{CP}, \mathrm{CO}-\mathrm{P}$ and $\mathrm{CO}-\mathrm{CP}$ ) than in the groups without Pg-LPS (C, C-C, $\mathrm{CO}$ and $\mathrm{CO}-\mathrm{C}$, respectively). In addition, the expression of those calcification-related factors in group $\mathrm{CO}$ was slightly higher than that in group $\mathrm{C}$, but the difference was not significant. The calcification effect in each co-culture group (CO-P, CO-C and CO-CP) was higher than that in each monoculture group (C-P, C-C and C-CP, respectively; Fig. 5).
Effects of Pg-LPS on calcified nodule formation by HUASMCs in the co-culture system. The cells were co-cultured with Pg-LPS and calcification-inducing medium and then stained with alizarin red $\mathrm{S}$ to detect the formation of calcified nodules. In the inverted phase-contrast microscopy images, the calcified nodules were scattered in the C-P and CO-P groups and were almost absent in the $\mathrm{C}$ and $\mathrm{CO}$ groups. All of the groups under calcification induction or calcification induction + Pg-LPS $(1 \mu \mathrm{g} / \mathrm{ml})$ conditions exhibited many calcified nodules, whilst the co-cultured $\mathrm{CO}-\mathrm{C}$ and $\mathrm{CO}-\mathrm{CP}$ groups had slightly more calcified nodules than those cultured in DMEM medium (Fig. 6).

The quantitative detection of calcified nodules indicated that calcium nodules formation in the group with general 
A
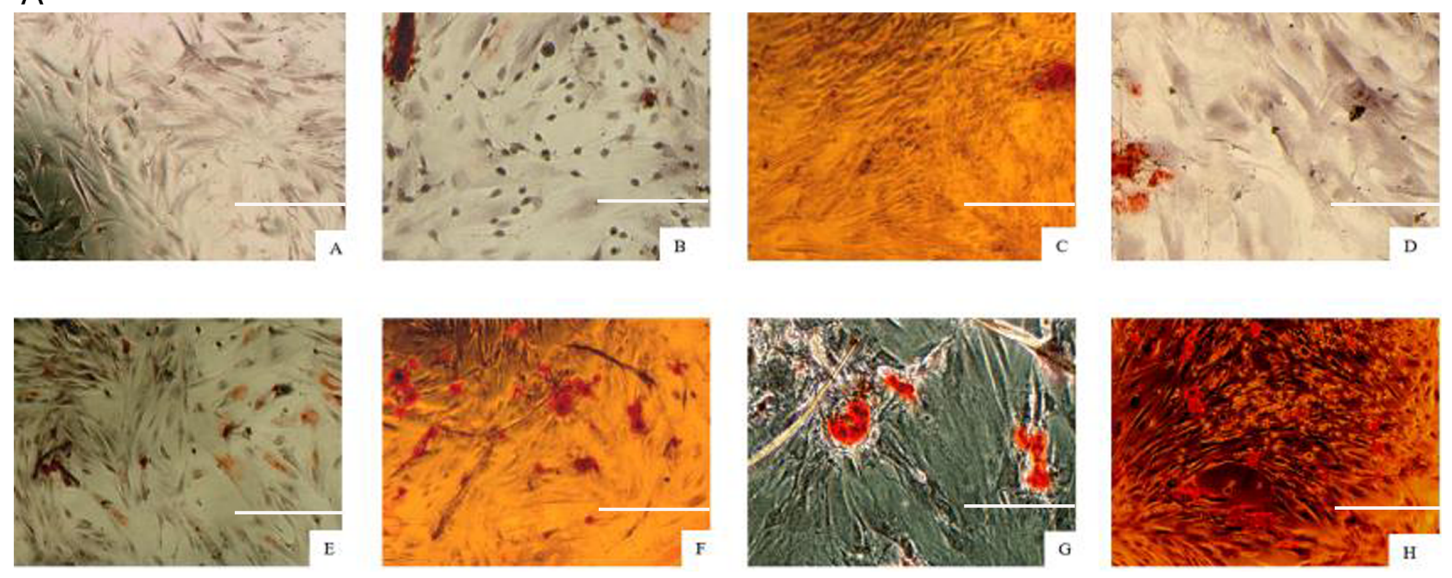

B
\#

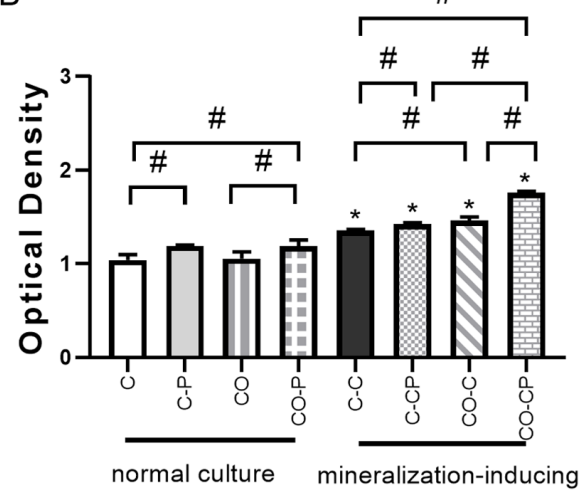

C: HUASMCs

C-P: HUASMCs+ Pg-LPS

CO: PDLCs-HUASMCs

CO-P:PDLCs-HUASMCs+Pg-LPS

C-C: HUASMCs+calcification-inducing culture medium

C-CPHUASMCs+ Pg-LPS+calcification-inducing culture medium

CO-C: PDLCs-HUASMCs+calcification-inducing culture medium

CO-CP: PDLCs-HUASMCs+Pg-LPS+calcification-inducing culture

medium

Figure 6. Effect of Pg-LPS on calcified nodule formation of HUASMCs in different groups. (AA) HUASMCs (group C); (AB) HUASMCs + Pg-LPS (group C-P); (AC) PDLCs-HUASMCs (group CO); (AD) PDLCs-HUASMCs + Pg-LPS (group CO-P); (AE) HUASMCs in calcification-inducing culture medium (group C-C); (AF) HUASMCs + Pg-LPS in calcification-inducing culture medium (group C-CP); (AG) PDLCs-HUASMCs in calcification-inducing culture medium (group CO-C); and (AH) PDLCs-HUASMCs + Pg-LPS in calcification-inducing culture medium (group CO-CP). The calcified nodules were scattered in the C-P and CO-P groups and were almost absent in the $\mathrm{C}$ and $\mathrm{CO}$ groups. All of the groups under calcification induction or calcification induction + Pg-LPS $(1 \mu \mathrm{g} / \mathrm{ml})$ conditions exhibited many calcified nodules, whilst the co-cultured CO-C and CO-CP groups had slightly more calcified nodules than those cultured in DMEM medium (scale bar, $100 \mu \mathrm{m}$ ). The quantified results of calcification based on alizarin red S staining three weeks after calcification induction (B). The calcified nodule formation was significantly higher in calcification-inducing medium and co-culture groups treated with Pg-LPS (1 $\mu \mathrm{g} / \mathrm{ml})$ than in the control groups. Values are expressed as the mean \pm standard deviation $(n=3) .{ }^{*} \mathrm{P}<0.05$, calcification-inducing culture medium vs. normal culture medium (i.e. group C-C vs. group C; group C-CP vs. group C-P; group CO-C vs. group CO; group CO-CP vs. group CO-P); " $\mathrm{P}<0.05$. HUASMCs, human umbilical artery smooth muscle cells; PDLCs, periodontal ligament cells; Pg-LPS, Porphyromonas gingivalis lipopolysaccharide..

culture in DMEM medium and Pg-LPS was more extensive than that in the corresponding control group. Each calcification induction group exhibited significantly more calcium nodule formation than the corresponding general culture group in DMEM medium. In the normal culture medium, the extent of calcium nodule formation by HUASMCs in group C and group $\mathrm{CO}$ was not significantly different. However, under the calcification induction conditions, calcium nodule formation in group CO-C was more extensive than in group $\mathrm{C}-\mathrm{C}$, whilst the largest amount of calcium nodules was detected in the CO-CP group (Fig. 6).

\section{Discussion}

Vascular calcification is a common pathological process of aging and various conditions including atherosclerotic coronary artery disease and diabetes. As one of the primary risk factors and early signs of cardiovascular disease, it mainly includes intima and tunica media calcification, both of which frequently occur at the same time. VSMCs were identified as the major cell type involved in the process of vascular calcification, possessing the ability to differentiate into osteoblast-like phenotype under the stimulation of pathological factors, and to synthesize and secrete related proteins and transcription factors promoting bone formation, as well as to express active $\operatorname{ALP}(21,22)$. The specific phenotypic transformation mechanism in VSMCs has remained to be fully elucidated, but inflammation and immune response may have an important role (23).

A comprehensive study suggested that periodontitis has a particular role in promoting vascular calcification of coronary heart disease, and coronary heart disease, in turn, aggravates the destruction of periodontal tissue to a certain extent (24-28). As one of the major pathogenic bacteria in periodontitis, Pg has a higher detection rate in the atherosclerotic plaques from patients with severe periodontitis and coronary heart disease than in patients with mild periodontitis and coronary heart disease. Yang et al (28) reported that Pg induced VSMC calcification and activity in mice and directly stimulated in vitro cultured aortic vessels to cause vascular 
wall calcinosis. Pg-LPS is an important pathogenic factor of bacteria. Han et al (29) demonstrated that low concentrations $(<100 \mu \mathrm{g} / \mathrm{ml})$ of Escherichia coli LPS promoted the abnormal proliferation of VSMCs in rats. A previous study by our group suggested that one day of culture with Pg-LPS at each concentration between $1 \mathrm{ng} / \mathrm{ml}$ and $\mu \mathrm{g} / \mathrm{ml}$ was able to promote the abnormal proliferation and ALP activity of VSMCs, affecting the expression of calcification-related genes (Runx2, ALP, BSP and OPN) (13). These results indicated that Pg-LPS affected VSMC calcification and provided indirect evidence of Pg-LPS promoting vascular tube calcification. Since the body's internal environment is complex, the effect of Pg-LPS on VSMCs in vivo requires further study.

At present, co-culture is a commonly used cell biology technique (30,31). Cell co-culture using Transwell inserts is able to achieve the interaction effect of cells in the upper and lower culture medium through a porous membrane, which more fully simulates the in vivo environment. In the present experiments, HUASMCs were selected as a cell model, and they were more convincing in the study of the relationship between human periodontitis and cardiovascular disease than the rat VSMCs used in the past (13). The co-culture system model of HPDLCs and HUASMCs using a Transwell chamber was selected to simulate the coexistence of periodontitis and vascular calcification in vivo. Of note, the morphology and growth rate of the two types of cells in the co-culture system did not change significantly compared with HUASMCs in monoculture, and this model more fully simulated the environment in vivo. Pg-LPS is an independent factor in periodontal disease and the co-culture system was able to simulate the environment of periodontal disease more realistically. It was reported that Pg-LPS stimulated HPDLCs to secrete IL-6, IL-8, TGF and other inflammatory factors (32-34). Thammasitboon et al (35) indicated that macrophages had an important role in LPS-induced osteoblast and PDLC apoptosis. Therefore, it was speculated in the present study that one of the reasons of co-cultured HUASMCs exhibiting abnormal proliferation was due to the effect of the HPDLCs. Periodontitis may promote the abnormal proliferation of VSMCs, whereby the abnormal proliferation of VSMCs may result in the formation of new intima, arteriosclerosis and restenosis $(21,36)$.

The expression of calcification-related genes was detected in the present study to further investigate the effect of Pg-LPS on HUASMC calcification. ALP is an early differentiation marker of osteoblasts and one of the confirmed phenotypic markers of osteoblast-like cells. It is also indispensable for osteoblast activity and bone formation (37). BSP is one of the acid glycoproteins secreted mainly by osteoblasts. It is able to activate osteoblasts or guide them to promote calcification. Its expression is an essential marker of calcification and matrix deposition (38). Runx2 is the initiation factor regulating the differentiation of undifferentiated mesenchymal stem cells into osteoblasts. Studies have indicated that the cranial cells of Runx $2^{-/-}$mice are not able to differentiate into osteoblasts when cultured in vitro (39). Runx2 is also a key transcription factor regulating the directional differentiation of osteoblasts and chondrocytes and has a critical regulatory role in calcification. The expression of Runx 2 in the atherosclerotic plaque is considered a sign for the initiation of vascular calcification $(13,40)$.
The present study suggested that ALP activity in HUASMCs increased after the action of Pg-LPS for $48 \mathrm{~h}$, and the ALP activity of HUASMCs in the co-culture group was stronger than that in the monoculture group. Pg-LPS and calcification-inducing medium promoted the expression of ALP, BSP and Runx 2 in HUASMCs, and the calcification-inducing medium had a more obvious promoting effect. In addition, when both factors were combined, the promotion effect was the strongest. This was similar to a previous study by our group, where Pg-LPS promoted the abnormal proliferation and ALP activity of VSMCs and affected the expression of calcification-related genes (Cbf $\alpha$ 1, ALP, BSP and OPN). Yin et al (41) indicated that LPS stimulated VSMC proliferation via phenotypic modulation and LPS promoted VSMC proliferation through the Toll-like receptor 4/Rac family small GTPase 1/Akt signaling pathway. ALP promotes ossification by dephosphorylation, destroying mineralization inhibitors and acting as a calcium-binding protein or phosphate transporter. Higher ALP activity suggests ossification and transformation (42). In addition, the promoting effect of Pg-LPS and calcification-inducing solution in the co-culture group was significantly higher than that in the monoculture group. It may be speculated that HPDLCs have a synergistic effect with Pg-LPS. HPDLCs may secrete certain inflammatory factors under the effect of Pg-LPS and aggravate the calcification of SMCs, and the calcification effect of co-culture HUASMCs was not only due to Pg-LPS but also because of the effect of HPDLCs. HPDLCs were able to promote the differentiation of HUASMCs into the osteoblast-like phenotype under the stimulation of Pg-LPS, indicating that vascular calcification may be induced and aggravated by periodontitis. Further studies by our group will examine the specific regulatory mechanism of LPS on ALP activity and the expression of calcification-related factors.

Alizarin red S staining is an effective method for determining the formation of calcified nodules. After 21 days of cell culture, calcium nodule staining and quantitative analysis indicated that both Pg-LPS and calcification-inducing solution promoted the formation of calcified nodules. Regarding the groups cultured in calcification-inducing medium, Pg-LPS increased the calcium nodules in the co-culture group compared to the HUASMCs in monoculture. These results indicated that Pg-LPS was able to promote HUASMC calcification, and in the presence of Pg-LPS and the calcification-inducing medium, the co-culture system had a more significant effect to stimulate HUASMC calcification. These results also confirmed that HPDLCs had a specific role in promoting HUASMC calcification under the effect of Pg-LPS.

In the present study, under the inflammatory conditions due to Pg-LPS stimulation, the proliferation and ALP activity of HUASMCs in the co-culture group increased significantly and the expression of calcification-related genes (ALP, Runx 2 and BSP) was also higher compared to the HUASMCs in monoculture. The high immune activity of Pg-LPS may explain these results. It changed the survival micro-environment of the two cell types and caused them to produce various inflammatory factors, with a synergistic role of the pro-inflammatory factors, aggravating the calcification of HUASMCs. The lack of western blot analysis being performed in the present study to investigate protein expression was a limitation of the present study 
and future studies should perform this to verify the present results. However, the human body is a complex organism and the growth and reproduction of cells are affected by numerous factors, such as the microenvironment and intercellular interactions. Therefore, the in vitro culture environment differed significantly from the body's internal environment. The in vitro experiments alone were not able to fully explain the mechanisms and signaling pathways responsible for the effect of Pg-LPS on VSMCs, necessitating further studies. The co-culture system was used to simulate the environment in vitro, which has the advantage of laying a better foundation for subsequent in vivo experiments compared with single-cell culture in vitro. Our group also considers constructing a better co-culture system or animal model to study the relationship of periodontitis and vascular calcification. The co-culture system may have an important role to stimulate HPDLCs to secrete IL-6, IL-8, TGF and other inflammatory factors. In the future, a better co-culture system may be established to study the relationship of inflammation factors with Pg-LPS in terms of protein and RNA expression.

In conclusion, Pg-LPS was able to induce the proliferation of HUASMCs and its effect was more significant in the HPDLCs-HUASMC co-culture system. Pg-LPS may stimulate calcification by increasing the ALP activity of HUASMCs and upregulating the expression of its calcification-related genes (ALP, Runx2 and BSP). This effect was greater in the co-culture system. The present results also indirectly demonstrated that eliminating inflammation may be an influential factor to inhibit vascular calcification.

\section{Acknowledgements}

Not applicable.

\section{Funding}

This work was supported by the National Natural Science Foundation of China (grant no. 81100755), the Health Department of Shandong Province (grant no. 2016WS0252) and the Key Laboratory of Stomatology and Biomedicine of Shandong Province (grant no. SDKQ201401).

\section{Availability of data and materials}

The datasets used and/or analyzed during the current study are available from the corresponding author on reasonable request.

\section{Authors' contributions}

JL, SS and GL contributed equally to the study design, manuscript preparation and data collection. WS, PS and WJ made contributions to the study design and data acquisition and drafted the manuscript. JD contributed to the study conception and critically revised the manuscript. KP supervised the research, oversaw the collection of results and data interpretation and critically revised the manuscript. JL and KP check and approve the authenticity of the raw data. All authors read and approved the final manuscript as submitted and agree to be accountable for all aspects of the work.

\section{Ethics approval and consent to participate}

The present study was approved by the Medical Ethics Committee of the Affiliated Hospital of Qingdao University (Qingdao, China; no. QYFYWZLL26135). Written informed consent was obtained from each patient or from the parents/ guardians of those participants who were minors.

\section{Patient consent for publication}

Not applicable.

\section{Competing interests}

The authors declare that they have no competing interests.

\section{References}

1. Safi IN, Mohammed Ali Hussein B and Al-Shammari AM: In vitro periodontal ligament cell expansion by co-culture method and formation of multi-layered periodontal ligament-derived cell sheets. Regen Ther 11: 225-239, 2019.

2. Gorasia DG, Glew MD, Veith PD and Reynolds EC: Quantitative proteomic analysis of the type IX secretion system mutants in Porphyromonas gingivalis. Mol Oral Microbiol 35: 78-84, 2020.

3. MaldonadoRF,Sá-Correia Iand ValvanoMA:Lipopolysaccharide modification in Gram-negative bacteria during chronic infection. FEMS Microbiol Rev 40: 480-493, 2016.

4. Le Sage F, Meilhac O and Gonthier MP: Porphyromonas gingivalis lipopolysaccharide induces pro-inflammatory adipokine secretion and oxidative stress by regulating Toll-like receptor-mediated signaling pathways and redox enzymes in adipocytes. Mol Cell Endocrinol 446: 102-110, 2017.

5. Zhao M, Dai W, Wang H, Xue C, Feng J, He Y, Wang P, Li S, Bai D and Shu R: Periodontal ligament fibroblasts regulate osteoblasts by exosome secretion induced by inflammatory stimuli. Arch Oral Biol 105: 27-34, 2019.

6. Naruishi K: Carotenoids and periodontal infection. Nutrients 12: $269,2020$.

7. Groeger S, Jarzina F, Windhorst A and Meyle J: Influence of retinoic acid on human gingival epithelial barriers. J Periodontal Res 51: 748-757, 2016.

8. Ehara S and Yoshiyama M: Quantitative analyses of coronary artery calcification by using clinical cardiovascular imaging. Clin Calcium 20: 1686-1692,2010.

9. Kendrick J and Chonchol M: The role of phosphorus in the development and progression of vascular calcification. Am J Kidney Dis 58: 826-834, 2011.

10. Lanzer P, Boehm M, Sorribas V, Thiriet M, Janzen J, Zeller T, St Hilaire C and Shanahan C: Medial vascular calcification revisited: Review and perspectives. Eur Heart J 35: 1515-1525, 2014.

11. Bessueille L and Magne D: Inflammation: A culprit for vascular calcification in atherosclerosis and diabetes. Cell Mol Life Sci 72: 2475-2489, 2015.

12. Ruiz JL, Hutcheson JD and Aikawa E: Cardiovascular calcification: current controversies and novel concepts. Cardiovasc Pathol 24: 207-212, 2015.

13. Liu G, Deng J, Zhang Q, Song W, Chen S, Lou X, Zhang P and Pan K: Porphyromonas gingivalis Lipopolysaccharide stimulation of vascular smooth muscle cells activates proliferation and calcification. J Periodontol 87: 828-836, 2016.

14. Li S, Fan SX and McKenna TM: Vascular smooth muscle cells on Matrigel as a model for LPS-induced hypocontractility and NO formation. Am J Physiol 272: H576-H584, 1997.

15. Tang XL and Meng HX: The inhibitory effect of calcitriol on the proliferation of hPDLCs populations of different vitamin D receptor Fok I genotypes. Shanghai Kou Qiang Yi Xue 18: 422-426, 2009 (In Chinese).

16. Dong X, Wang YS, Dou GR, Hou HY, Shi YY, Zhang R, Ma K, Wu L, Yao LB, Cai Y, et al: Influence of Dll4 via HIF-1 $\alpha$-VEGF signaling on the angiogenesis of choroidal neovascularization under hypoxic conditions. PLoS One 6: e18481, 2011. 
17. Wang YL, Pan KQ, Sun Y and Deng J: Effect of lipopolysaccharide on the expression of ALP, BSP, DSPP in rat dental pulp cells. Shanghai Kou Qiang Yi Xue 23: 431-435, 2014 (In Chinese).

18. Young N, Mikhalkevich N and Yan Y, Chen D and Zheng WP: Differential regulation of osteoblast activity by $\mathrm{Th}$ cell subsets mediated by parathyroid hormone and IFN-gamma J Immunol 175: 8287-8295, 2005.

19. Aonuma H, Ogura N, Takahashi K, Fujimoto Y, Iwai S, Hashimoto H, Ito K, Kamino Y and Kondoh T: Characteristics and osteogenic differentiation of stem/progenitor cells in the human dental follicle analyzed by gene expression profiling. Cell Tissue Res 350: 317-331, 2012.

20. Gregory CA, Gunn WG, Peister A and Prockop DJ: An Alizarin red-based assay of mineralization by adherent cells in culture: Comparison with cetylpyridinium chloride extraction. Anal Biochem 329: 77-84, 2004

21. Liberman M, Pesaro AE, Carmo LS and Serrano CV Jr: Vascular calcification: Pathophysiology and clinical implications. Einstein (Sao Paulo) 11: 376-382, 2013.

22. Burton DG, Matsubara H and Ikeda K: Pathophysiology of vascular calcification: Pivotal role of cellular senescence in vascular smooth muscle cells. Exp Gerontol 45: 819-824, 2010.

23. Davis-Dusenbery $\mathrm{BN}, \mathrm{Wu} \mathrm{C}$ and Hata A: Micromanaging vascular smooth muscle cell differentiation and phenotypic modulation. Arterioscler Thromb Vasc Biol 31: 2370-2377, 2011

24. Bahekar AA, Singh S, Saha S, Molnar J and Arora R: The prevalence and incidence of coronary heart disease is significantly increased in periodontitis: A meta-analysis. Am Heart J 154: 830-837, 2007.

25. Humphrey LL, Fu R, Buckley DI, Freeman M and Helfand M: Periodontal disease and coronary heart disease incidence: $\mathrm{A}$ systematic review and meta-analysis. J Gen Intern Med 23 2079-2086, 2008.

26. Khader YS, Albashaireh ZS and Alomari MA: Periodontal diseases and the risk of coronary heart and cerebrovascular diseases: A meta-analysis. J Periodontol 75: 1046-1053, 2004.

27. Zhang YM, Zhong LJ, He BX, Nie J, Wang X and Li WC: Study on the correlation between coronary heart disease and chronic periodontitis. Zhonghua Liu Xing Bing Xue Za Zhi 27: 256-259, 2006 (In Chinese)

28. Yang WW, Guo B, Jia WY and Jia Y: Porphyromonas gingiva lis-derived outer membrane vesicles promote calcification of vascular smooth muscle cells through ERK1/2-RUNX2. FEBS Open Bio 6: 1310-1319, 2016

29. Han F, Hou N, Liu Y, Huang N, Pan R, Zhang X, Mao E and Sun $X$ : Liraglutide improves vascular dysfunction by regulating a cAMP-independent PKA-AMPK pathway in perivascular adipose tissue in obese mice. Biomed Pharmacother 120: 109537, 2019.

30. Gharaibeh B, Lu A, Tebbets J, Zheng B, Feduska J, Crisan M, Péault B, Cummins J and Huard J: Isolation of a slowly adhering cell fraction containing stem cells from murine skeletal muscle by the preplate technique. Nat Protoc 3: 1501-1509, 2008.
31. Saldarriaga Fernández IC, Busscher HJ, Metzger SW, Grainger DW and van der Mei HC: Competitive time- and density-dependent adhesion of staphylococci and osteoblasts on crosslinked poly(ethylene glycol)-based polymer coatings in co-culture flow chambers. Biomaterials 32: 979-984, 2011.

32. Sun Y, Shu R, Li CL and Zhang MZ: Gram-negative periodontal bacteria induce the activation of Toll-like receptors 2 and 4 , and cytokine production in human periodontal ligament cells. J Periodontol 81: 1488-1496, 2010.

33. Tintut Y, Patel J, Parhami F and Demer LL: Tumor necrosis factor-alpha promotes in vitro calcification of vascular cells via the cAMP pathway. Circulation 102: 2636-2642, 2000.

34. Yamamoto T, Kita M, Oseko F, Nakamura T, Imanishi J and Kanamura N: Cytokine production in human periodontal ligament cells stimulated with Porphyromonas gingivalis. J Periodontal Res 41: 554-559, 2006.

35. Thammasitboon K, Goldring SR and Boch JA: Role of macrophages in LPS-induced osteoblast and PDL cell apoptosis. Bone 38: 845-852, 2006

36. Sadowitz B, Seymour K, Gahtan V and Maier KG: The role of hyaluronic acid in atherosclerosis and intimal hyperplasia. J Surg Res 173: e63-e72, 2012.

37. Boström KI, Rajamannan NM and Towler DA: The regulation of valvular and vascular sclerosis by osteogenic morphogens. Circ Res 109: 564-577, 2011.

38. Khan SZ, Kokubu E, Matsuzaka K and Inoue T: Behaviour of rat-cultured dental pulp cells in three-dimensional collagen type-1 gel in vitro and in vivo. Aust Endod J 39: 137-145, 2013.

39. Ciceri P, Elli F, Cappelletti L, Tosi D, Savi F, Bulfamante G and Cozzolino M: Osteonectin (SPARC) expression in vascular calcification: In vitro and ex vivo studies. Calcif Tissue Int 99: 472-480, 2016.

40. Pang J, Zhang Y, Ke J, Yu Q, He W and Wu B: Upregulation of dentin matrix protein 1 promoter activities by core binding factor alpha1 in human dental pulp stem cells. Biochem Biophys Res Commun 357: 505-510, 2007.

41. Yin Q, Jiang D, Li L, Yang Y, Wu P, Luo Y, Yang R and Li D: LPS promotes vascular smooth muscle cells proliferation through the TLR4/Rac1/Akt signalling pathway. Cell Physiol Biochem 44: 2189-2200, 2017.

42. Lv X-C, Bi L-J, Jiang Y and Wang X: Effects of icariin on the alkline phosphatase activity of human periodontal ligament cells inhibited by lipopolysaccharide. Mol Med Rep 8: 1411-1415, 2013.

This work is licensed under a Creative Commons Attribution-NonCommercial-NoDerivatives 4.0 International (CC BY-NC-ND 4.0) License. 